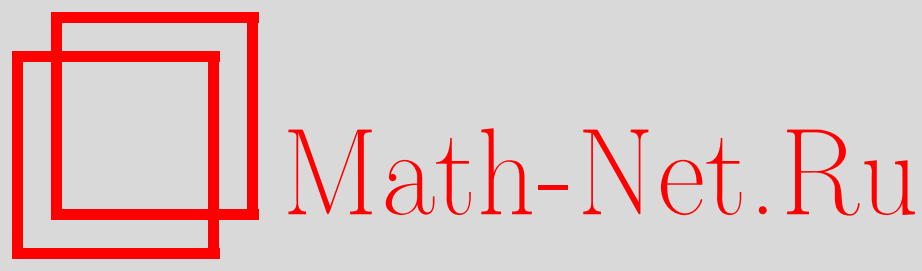

А. К. Погребков, Высшие разностные уравнения Хироты и их редукции, ТМФ, 2018, том 197, номер 3, 444-463

DOI: https://doi.org/10.4213/tmf9569

Использование Общероссийского математического портала Math-Net.Ru подразумевает, что вы прочитали и согласны с пользовательским соглашением http: //www . mathnet.ru/rus/agreement

Параметры загрузки:

IP : 54.198 .55 .26

26 апреля 2023 г., 13:24:22

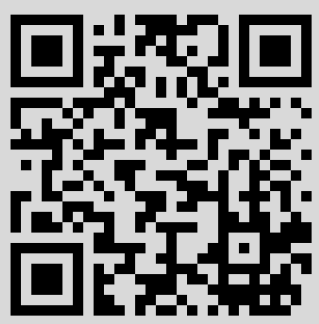




\title{
ФИЗИКА
}

Том 197, № 3

декабрь, 2018

(C) 2018 г.

А. К. Погребков*

\section{ВЫСШИЕ РАЗНОСТНЫЕ УРАВНЕНИЯ ХИРОТЫ И ИХ РЕДУКЦИИ}

\begin{abstract}
Ранее нами был предложен подход к построению интегрируемых уравнений, основанный на динамике в ассоциативных алгебрах, заданной коммутаторными соотношениями. В рамках этого подхода уравнения эволюции, определенные посредством коммутаторов (или преобразований подобия) с функциями от одного и того же оператора, по построению совместны, и, как результат, возникают линейные уравнения, на основе которых с помощью специальной процедуры одевания строятся нелинейные интегрируемые уравнения и соответствующие пары Лакса. Предлагается развитие данного подхода, основанное на введении высших аналогов знаменитого разностного уравнения Хироты. Также рассмотрены некоторые $(1+1)$-мерные разностные интегрируемые уравнения, которые возникают как редукции либо самого́ разностного уравнения Хироты, либо высшего уравнения его иерархии.
\end{abstract}

Ключевые слова: интегрируемость, коммутаторные тождества, разностное уравнение Хироты, высшие интегрируемые уравнения, редукции.

DOI: https://doi.org/10.4213/tmf9569

Памяти Людвига Дмитриевича Фаддеева

\section{1. ВВЕДЕНИЕ}

В данной статье мы вводим и исследуем высшие интегрируемые обобщения знаменитого разностного уравнения Хироты (РУХ). Оно было введено в работах [1], [2] в билинейной форме как уравнение на $\tau$-функцию

$$
\tau^{(1)}(m) \tau^{(2,3)}(m)+\tau^{(2)}(m) \tau^{(3,1)}(m)+\tau^{(3)}(m) \tau^{(1,2)}(m)=0,
$$

где $\tau(m)=\tau\left(m_{1}, m_{2}, m_{3}\right)$ - функция, зависящая от трех целых чисел (независимых переменных), $m_{1}, m_{2}, m_{3} \in \mathbb{Z}$. Здесь и далее верхние индексы 1,2 и 3 в скобках

Исследование выполнено за счет гранта Российского научного фонда (проект № 14-50-00005).

* Математический институт им. В. А. Стеклова Российской академии наук, Москва, Россия. E-mail: pogreb@mi-ras.ru 
означают единичный сдвиг переменной с тем же номером:

$$
\begin{aligned}
\tau^{(1)}(m)= & \tau\left(m_{1}+1, m_{2}, m_{3}\right), \quad \tau^{(2)}(m)=\tau\left(m_{1}, m_{2}+1, m_{3}\right), \\
& \tau^{(2,3)}(m)=\tau\left(m_{1}, m_{2}+1, m_{3}+1\right) \quad \text { и т. д. }
\end{aligned}
$$

РУХ является $(2+1)$-мерным интегрируемым уравнением, которому посвящено множество статей (см., например, [2]-[11] и цитированную в них литературу), поскольку оно порождает многие известные интегрируемые дифференциальные уравнения в частных производных, дифференциально-разностные и разностные уравнения как различные непрерывные пределы и $(1+1)$-мерные интегрируемые редукции. В частности, к таким уравнениям относятся обычные и модифицированные уравнения Кортевега-де Фриза (КдФ) и Кадомцева-Петвиашвили, двумеризованная система Тоды, уравнения синус-Гордона, Бенджамина-Оно и т. д. Поэтому РУХ часто рассматривается как фундаментальная интегрируемая система.

Хорошо известным инструментом исследования нелинейных интегрируемых уравнений является $\tau$-функция, но при изучении прямой и обратной задач рассеяния она неудобна. Поэтому в литературе (см., скажем, работу [6]) была введена функция $v(m)=v\left(m_{1}, m_{2}, m_{3}\right)$, заданная равенствами

$$
\begin{aligned}
& v^{(1)}(m)-v^{(3)}(m)=\frac{\tau^{(1,3)}(m) \tau(m)}{\tau^{(1)}(m) \tau^{(3)}(m)}, \\
& v^{(2)}(m)-v^{(1)}(m)=\frac{\tau^{(1,2)}(m) \tau(m)}{\tau^{(2)}(m) \tau^{(1)}(m)},
\end{aligned}
$$

которая позволяет переписать (1.1) в виде (см. обозначения (1.2))

$$
v^{(1,2)}\left(v^{(1)}-v^{(2)}\right)+v^{(2,3)}\left(v^{(2)}-v^{(3)}\right)+v^{(3,1)}\left(v^{(3)}-v^{(1)}\right)=0 .
$$

В этих терминах пара Лакса для РУХ дается любыми двумя из трех уравнений

$$
\begin{aligned}
& \varphi^{(2)}=\varphi^{(1)}+\left(v^{(2)}-v^{(1)}\right) \varphi, \\
& \varphi^{(3)}=\varphi^{(2)}+\left(v^{(3)}-v^{(2)}\right) \varphi, \\
& \varphi^{(1)}=\varphi^{(3)}+\left(v^{(1)}-v^{(3)}\right) \varphi,
\end{aligned}
$$

которые подчеркивают симметрию РУХ по всем трем переменным $m_{i}$. В то же время уравнение (1.5) можно рассматривать как эволюционное уравнение, где, скажем, $m_{1}$ и $m_{2}$ играют роль пространственных переменных, а $m_{3}$ - временно́й.

В нашей работе при выводе высших обобщений РУХ мы следуем работам [12]-[14], в которых нелинейные уравнения выводятся как результат специальной процедуры одевания соответствующих линейных уравнений. Таким образом, начальная точка нашего подхода - это вывод таких линейных уравнений, в каком-то смысле являющихся уравнениями на данные рассеяния. Это достигается с помощью следующей конструкции. Пусть $A$ и $B$ - два произвольных элемента некоторой ассоциативной алгебры над $\mathbb{C}$. Введем зависимость элемента $B$ от дискретных или непрерывных параметров посредством коммутаторов некоторых функций от $A$ с $B$. Мы рассматриваем термин "коммутатор" в обоих смыслах: алгебраическом для непрерывных параметров или групповом (как преобразования подобия) для дискретного случая. 
Эти параметры интерпретируются как независимые переменные (времена), поскольку они задают взаимно коммутирующие потоки на алгебре, соответственно непрерывные или дискретные. Тогда линейное уравнение получается из коммутаторного тождества, специфического для выбранных функций от элемента $A$ и справедливого на произвольной ассоциативной алгебре.

Статья организована следующим образом. В разделе 2 мы используем пример PУХ для демонстрации основных шагов нашего подхода (подробности см. в [13]). В разделе 3 мы рассматриваем некоторые редукции этой $(2+1)$-мерной интегрируемой системы к $(1+1)$-интегрируемым системам. В разделе 4 мы приводим общий метод построения высших уравнений типа РУХ, включая разностный аналог системы Захарова-Шабата. В разделе 5 рассматривается пример такого высшего РУХ. Некоторые $(1+1)$-мерные редукции этого уравнения даны в разделе 6 . Заключительные замечания приведены в разделе 7 .

\section{2. РУХ КАК ПРИМЕР ОБЩЕЙ КОНСТРУКЦИИ}

\section{1. Коммутаторное тождество и линейное уравнение на ассоциативной} алгебре. Здесь мы следуем работе [13], чтобы проиллюстрировать детали нашего подхода, и начинаем с простейшего случая самого́ РУХ. Пусть дана ассоциативная алгебра с единицей $I$ и для некоторого элемента $A$ в этой алгебре существуют обратные элементы $\left(A-a_{i} I\right)^{-1}$ при некоторых постянных $a_{1}, a_{2}, a_{3} \in \mathbb{C}$ (где $\left.a_{1} \neq a_{2} \neq a_{3} \neq a_{1}\right)$. Пусть $B$ - еще один элемент этой алгебры. Введем зависимость элемента $B$ от трех разностных переменных $m_{1}, m_{2}, m_{3}$, принадлежащих $\mathbb{Z}$, как (см. обозначения (1.2))

$$
\begin{aligned}
& B^{(1)}=\left(A-a_{1} I\right) B\left(A-a_{1} I\right)^{-1}, \\
& B^{(2)}=\left(A-a_{2} I\right) B\left(A-a_{2} I\right)^{-1}, \\
& B^{(3)}=\left(A-a_{3} I\right) B\left(A-a_{3} I\right)^{-1} .
\end{aligned}
$$

Тогда $B(m)$ удовлетворяет линейному дифференциальному уравнению

$$
a_{12}\left\{B^{(12)}+B^{(3)}\right\}+\operatorname{cycle}\{1,2,3\}=0,
$$

где разности

$$
a_{i j}=a_{i}-a_{j}
$$

отличны от нуля. Уравнение (2.2) следует из коммутаторного тождества

$$
\begin{aligned}
& a_{12}\left\{\left(A-a_{1}\right)\right.\left(A-a_{2}\right) B\left(A-a_{1}\right)^{-1}\left(A-a_{2}\right)^{-1}+ \\
&\left.+\left(A-a_{3}\right) B\left(A-a_{3}\right)^{-1}\right\}+\operatorname{cycle}(1,2,3)=0,
\end{aligned}
$$

справедливого на произвольной ассоциативной алгебре. Уравнение (2.2) есть линеаризованная версия РУХ (1.5) (см. формулу (2.27) ниже или работу [13]).

2.2. Операторная реализация и процедура одевания. В статье [13] для формулировки процедуры одевания мы использовали аналогию с псевдодифференциальными операторами. Рассмотрим (бесконечные) матрицы $F, G, \ldots$, пусть $T$ 
означает матрицу сдвига, $T_{m_{1}, m_{1}^{\prime}}=\delta_{m_{1}, m_{1}^{\prime}+1}$. Для любой матрицы $F=\left\{F_{i j}\right\}_{i, j \in \mathbb{Z}}$ положим $f_{n}\left(m_{1}\right)=F_{m_{1}, m_{1}-n}$, так что матрицу $F$ можно записать как

$$
F=\sum_{n \in \mathbb{Z}} f_{n} T^{n}
$$

где все матрицы $f_{n}$ диагональны, $f_{n}=\operatorname{diag}\left\{f_{n}\left(m_{1}\right)\right\}_{m_{1} \in \mathbb{Z}}$, т. е. попарно коммутируют. Заметим, что это только наводящие соображения, поэтому мы не обсуждаем сходимость выписанного выше ряда. С точностью до этого замечания мы однозначно сопоставляем каждой матрице $F$ ее символ

$$
\widetilde{F}\left(m_{1}, z\right)=\sum_{n \in \mathbb{Z}} f_{n}\left(m_{1}\right) z^{n}
$$

где $m_{1} \in \mathbb{Z}, z=z_{\Re}+i z_{\Im} \in \mathbb{C}$. Легко видеть, что стандартное произведение матриц $F$ и $G$ в терминах этих символов принимает вид

$$
\widetilde{F G}\left(m_{1}, z\right)=\oint_{|\zeta|=1} \frac{d \zeta}{2 \pi i \zeta} \widetilde{F}\left(m_{1}, z \zeta\right) \sum_{m_{1}^{\prime} \in \mathbb{Z}} \zeta^{m_{1}-m_{1}^{\prime}} \widetilde{G}\left(m_{1}^{\prime}, z\right) .
$$

В качестве полезных примеров отметим, что для единичной матрицы и матрицы сдвига ( $I$ и $T$ соответственно) $i_{n}\left(m_{1}\right)=\delta_{m_{1}, 0}$ и $t_{n}\left(m_{1}\right)=\delta_{m_{1}, 1}$, так что по предыдущему определению для символов мы имеем

$$
\widetilde{I}(m, z)=1, \quad \widetilde{T}(m, z)=z .
$$

Равенство (2.5) показывает, что мы используем аналог нормального произведения: все операторы сдвига помещаются справа от операторов умножения. В частности, пусть $F$ - диагональная матрица, что означает, что ее символ не зависит от $z$ : $\widetilde{F}\left(m_{1}, z\right) \equiv \widetilde{F}\left(m_{1}\right)$ (см. равенства $\left.(2.5),(2.6)\right)$. Тогда в силу $(2.7)$ для любого оператора $G$

$$
\widetilde{F G}\left(m_{1}, z\right)=\widetilde{F}\left(m_{1}\right) \widetilde{G}\left(m_{1}, z\right) .
$$

Таким образом, операторы с символами, не зависящими от $z$, играют роль операторов умножения. Напротив, пусть $G$ - функция только от оператора сдвига, т. е. в силу $(2.5),(2.6)$ ее символ не зависит от дискретной переменной, $\widetilde{G}\left(m_{1}, z\right) \equiv \widetilde{G}(z)$. Тогда с учетом $(2.7)$ для любого $F$ получаем

$$
\widetilde{F G}\left(m_{1}, z\right)=\widetilde{F}\left(m_{1}, z\right) \widetilde{G}(z) .
$$

Преобразование подобия с оператором $T$, как следует из $(2.7)$ и $(2.10)$, дает сдвиг дискретной переменной:

$$
\widetilde{T F T^{-1}}\left(m_{1}, z\right)=\widetilde{F}\left(m_{1}+1, z\right), \quad \text { т. е. } \quad T F T^{-1}=F^{(1)},
$$

где было использовано обозначение (1.2). Это соотношение существенно для последующих рассуждений.

Далее мы рассматриваем множество "псевдоматричных" операторов $F, G, \ldots$, заданных их символами $\widetilde{F}, \widetilde{G}, \ldots$ с указанным выше законом композиции. Мы налагаем условие, что эти символы являются обобщенными функциями умеренного роста 
по своим переменным или коэффициентами Фурье таких обобщенных функций. Но в общем случае мы не ожидаем никакой связи типа (2.5) этих операторов с матрицами, в частности мы не подразумеваем наличие каких-либо свойств аналитичности символов операторов по переменной $z$. Поэтому на данном множестве операторов можно ввести операции, которые хорошо определены посредством символов, но не имеют аналогов на пространстве матриц. В частности, в работе [13] мы определили операцию $\bar{\partial}$-дифференцирования $F \rightarrow \bar{\partial} F$ :

$$
(\widetilde{\bar{\partial} F})(m, z)=\frac{\partial \widetilde{F}(m, z)}{\partial \bar{z}} .
$$

Эта производная дает меру отклонения символа оператора от аналитичности, и потому она также дает меру отклонения оператора от бесконечной матрицы, т. е. от ситуации, когда ряд в (2.5) сходится. В частности, единичный оператор и оператор сдвига удовлетворяют, как следует из (2.8), уравнениям

$$
\bar{\partial} I=0, \quad \bar{\partial} T=0 .
$$

2.3. Процедура одевания. Мы рассматриваем операторы $A$ и $B$ как операторы указанного выше вида с символами $\widetilde{A}$ и $\widetilde{B}$. Зависимость символа оператора $B$ от $m_{1}, B^{(1)}=\left(A-a_{1}\right) B\left(A-a_{1}\right)^{-1}$, в точности такая же, как при преобразовании подобия (2.11) с оператором $T$. Таким образом, мы можем положить

$$
A=T+a_{1}, \quad \text { т. е. } \widetilde{A}(m, z)=z+a_{1} .
$$

Тогда в соответствии с $(2.1)$

$$
\begin{gathered}
B^{(1)}=T B T^{-1}, \quad B^{(2)}=\left(T+a_{12}\right) B\left(T+a_{12}\right)^{-1}, \\
B^{(3)}=\left(T+a_{13}\right) B\left(T+a_{13}\right)^{-1},
\end{gathered}
$$

где использованы обозначения (1.2) и (2.3). Теперь символ $\widetilde{B}\left(m_{1}, m_{2}, m_{3}, z\right)$ оператора $B$, помимо переменной $z$, зависит от дискретных переменных $m_{1}, m_{2}, m_{3} \in \mathbb{Z}$. В дальнейшем мы используем обозначение $\widetilde{B}(m, z)$, полагая $m=\left\{m_{1}, m_{2}, m_{3}\right\}$. Зависимость от $m_{2}$ и $m_{3}$ не нарушает равенство $(2.7)$, где произведение символов нужно понимать как поточечное по этим переменным, так что

$$
(F G)^{(i)}=F^{(i)} G^{(i)}, \quad i=1,2,3,
$$

причем для $i=1$ это равенство следует из (2.7).

С учетом указанной в (2.15) зависимости оператора $B$ от $m$ его символ можно представить как

$$
\widetilde{B}(m, z)=\oint_{|\zeta|=1} \frac{d \zeta}{2 \pi i \zeta} \zeta^{m_{1}}\left(\frac{z \zeta+a_{12}}{z+a_{12}}\right)^{m_{2}}\left(\frac{z \zeta+a_{13}}{z+a_{13}}\right)^{m_{3}} b(\zeta, z),
$$

где $b(\zeta, z)$ - некоторая функция. Как было показано в работе [13], чтобы избежать роста этого символа по переменным $m_{2}$ и $m_{3}$, достаточно положить все $a_{j}$ вещественными, а носитель функции $b(\zeta, z)$ считать сосредоточенным на поверхности $\zeta=\bar{z} / z$. Таким образом, для символа оператора $B$ мы получаем представление

$$
\widetilde{B}(m, z)=\left(\frac{\bar{z}}{z}\right)^{m_{1}}\left(\frac{\bar{z}+a_{12}}{z+a_{12}}\right)^{m_{2}}\left(\frac{\bar{z}+a_{13}}{z+a_{13}}\right)^{m_{3}} f(z),
$$

где $f(z)-$ произвольная функция от $z \in \mathbb{C}$. 
Основной объект нашей конструкции, оператор одевания $K$ с символом $\widetilde{K}(m, z)$, вводится как решение $\bar{\partial}$-задачи

$$
\bar{\partial} K=K B, \quad \lim _{z \rightarrow \infty} \tilde{K}(m, z)=1,
$$

где произведение в правой части понимается в смысле (2.7). Ниже мы предполагаем однозначную разрешимость этой задачи, что играет решающую роль в нашей конструкции, но несущественно для ее результатов (см. раздел 7). Зависимость оператора $K$ от переменных $m$ вводится посредством той же $\bar{\partial}$-задачи:

$$
\bar{\partial} K^{(j)}=K^{(j)} B^{(j)}, \quad \lim _{z \rightarrow \infty} \widetilde{K^{(j)}}(m, z)=1, \quad j=1,2,3,
$$

где мы учли равенство (2.16).

Совместность уравнений эволюции для оператора $B$ очевидна по построению. Тогда в силу (2.16) и (2.20) $\bar{\partial} K^{(i, j)}=K^{(i, j)} B^{(i, j)}$ и $\bar{\partial} K^{(j, i)}=K^{(j, i)} B^{(i, j)}$ для любых $i, j=1,2,3$. Таким образом, разность $K^{(i, j)}-K^{(j, i)}$ удовлетворяет $\bar{\partial}$-уравнению в (2.19), но с нулевой асимптотикой, так что эта разность обращается в ноль в силу предположения об однозначной разрешимости. Рассмотрим следствия уравнений (2.15) для оператора $K$. Заметим, что этот оператор, как и любой оператор из рассматриваемого класса, удовлетворяет равенству (2.11),

$$
K^{(1)}=T K T^{-1}
$$

что совместно с (2.20) при $j=1$ в силу первого равенства в (2.15). Рассмотрим теперь $j=2$. Благодаря (2.13) и (2.15) мы получаем

$$
\bar{\partial}\left(K^{(2)}\left(T+a_{12}\right)\right)=\left(K^{(2)}\left(T+a_{12}\right)\right) B,
$$

т. е. произведение $K^{(2)}\left(T+a_{12}\right)$ удовлетворяет тому же $\bar{\partial}$-уравнению, но с асимптотикой, растущей линейно на z-бесконечности. И снова в силу предположения об однозначной разрешимости задачи (2.19) существует оператор умножения $X$ - оператор с символом, не зависящим от переменной $z,-$ такой, что $K^{(2)}\left(T+a_{12}\right)=(T+X) K$. Уточняя асимптотическое поведение в (2.19) посредством следующего члена разложения,

$$
K=I+u T^{-1}+\cdots, \quad z \rightarrow \infty,
$$

где многоточие означает члены с символами, которые убывают быстрее, чем $z^{-1}$, а $u$-оператор умножения, получаем в силу $(2.21)$

$$
K^{(2)}\left(T+a_{12}\right)=K^{(1)} T+\left(a_{12}+u^{(2)}-u^{(1)}\right) K .
$$

Аналогичные рассуждения показывают, что эволюция по переменной $m_{3}$ дается уравнением

$$
K^{(3)}\left(T+a_{13}\right)=K^{(1)} T+\left(a_{13}+u^{(3)}-u^{(1)}\right) K .
$$

Вследствие соотношений (2.9) и (2.10) уравнения (2.22) и (2.23) в терминах символов имеют вид

$$
\begin{aligned}
& \left(z+a_{12}\right) \widetilde{K}^{(2)}(m, z)=z \widetilde{K}^{(1)}(m, z)+\left(v^{(2)}(m)-v^{(1)}(m)\right) \widetilde{K}(m, z) \\
& \left(z+a_{13}\right) \widetilde{K}^{(3)}(m, z)=z \widetilde{K}^{(1)}(m, z)+\left(v^{(3)}(m)-v^{(1)}(m)\right) \widetilde{K}(m, z)
\end{aligned}
$$


так что переменная $z \in \mathbb{C}$ играет роль спектрального параметра. Здесь мы ввели для краткости новую зависимую переменную

$$
v(m)=u(m)-m_{1} a_{1}-m_{2} a_{2}-m_{3} a_{3} .
$$

Уравнения (2.25) совместны по построению, и непосредственная проверка этой совместности дает, что функция $v(m)$ удовлетворяет (1.5), т. е. РУХ. При подстановке (2.26) это уравнение принимает вид

$$
u^{(12)}\left(u^{(2)}-u^{(1)}+a_{12}\right)+a_{12} u^{(3)}+\text { cycle }=0,
$$

так что исходное уравнение (2.2) есть его линеаризованная версия. Заметим, что хотя константы $a_{i}$ не входят в уравнение (1.5), они в силу (2.26) определяют асимптотическое поведение функции $v(m)$ : на бесконечности она растет линейно по $m$. В работе [13] мы показали, что это асимптотическое поведение решает проблему некорректности задачи Коши для уравнения (1.5).

Для перехода к стандартным обозначениям введем решение Йоста

$$
\varphi(m, z)=\widetilde{K}(m, z) z^{m_{1}}\left(z+a_{12}\right)^{m_{2}}\left(z+a_{13}\right)^{m_{3}}
$$

Тогда уравнения (2.25a) и (2.25б) сводятся к (1.6а) и (1.6в), т. е. к паре Лакса для РУХ. Решение Йоста также упрощает запись $\bar{\partial}$-задачи (2.19). Чтобы показать это, заметим что в силу (2.7) и (2.18) уравнение задачи (2.19) в терминах символа оператора одевания записывается как

$$
\frac{\partial \widetilde{K}(m, z)}{\partial \bar{z}}=\widetilde{K}(m, \bar{z})\left(\frac{\bar{z}}{z}\right)^{m_{1}}\left(\frac{\bar{z}+a_{12}}{z+a_{12}}\right)^{m_{2}}\left(\frac{\bar{z}+a_{13}}{z+a_{13}}\right)^{m_{3}} f(z)
$$

что в силу (2.28) дает стандартную $\bar{\partial}$-задачу с сопряжением:

$$
\frac{\partial \varphi(m, z)}{\partial \bar{z}}=\varphi(m, \bar{z}) f(z)
$$

Представление (2.29) показывает, что закон дисперсии вырожден; это свойство, как хорошо известно [15], имеет непосредственное отношение к интегрируемости.

\section{3. (1 + 1)-МЕРНЫЕ РЕДУКЦИИ РУХ}

В работах [12]-[14] и [16] мы показали, что подход, основанный на коммутаторных соотношениях, приводит к интегрируемым уравнениям в $(2+1)$ измерениях. Для получения $(1+1)$-мерных интегрируемых систем следует выполнить редукции. Следуя идее нашего подхода, мы начинаем с построения редукций линейного уравнения (2.2) для $B$, а затем применяем процедуру одевания для получения нелинейных интегрируемых систем. Таким образом, в данном случае размерная редукция понимается как соотношение между значениями оператора $B$, полученных действием некоторых сдвигов независимых переменных $m_{i}$. Такое соотношение должно быть совместно с (2.18) и должно сохранять зависимость оператора $B$ от двух независимых переменных. 
Благодаря представлению (2.18) легко видеть, что любая такая редукция приводит к уравнению для спектрального параметра $z$ : он должен принадлежать некоторой кривой на $\mathbb{C}$. Это возможно, только если функция $f(z)$ в $(2.18)$ и, следовательно, сам символ $\widetilde{B}(m, z)$ имеют носитель на этой кривой, что мы понимаем здесь для простоты как пропорциональность соответствующей дельта-функции. Но тогда $(2.29)$ означает, что символ $\widetilde{K}(m, z)$ является аналитической функцией вне этой кривой, поэтому обратная задача (2.19) должна быть заменена стандартной проблемой Римана-Гильберта. Мы не углубляемся здесь в эти детали, поскольку, если мы имеем редукцию в указанном выше смысле, редуцированную пару Лакса и нелинейную интегрируемую систему можно вывести непосредственно из соответствующих $(2+1)$-мерных уравнений.

Естественно полагать, что простейшая редукция РУХ задается как $B^{(i)}=B$ для некоторого $i$, т. е. условием независимости символа $\widetilde{B}(m, z)$ от одной из переменных $m_{1}, m_{2}, m_{3}$. Но представление (2.18) показывает, что тогда она не является редукцией в нашем смысле, поскольку она возможна, только если $z_{\Im}=0$, что делает символ $\widetilde{B}(m, z)$ не зависящим от всех остальных переменных. Поэтому, чтобы получить нетривиальные редукции, следует найти более сложные соотношения между независимыми переменными. Отметим, что тривиальное условие $z_{\Im}=0$ появляется при всех возможных редукциях, так что ниже мы его опускаем.

3.1. Редукция $B^{(2)}=B^{(-1)}$. Начнем с условия $B^{(2)}=B^{(-1)}$, где $(-1)$ в верхнем индексе означает сдвиг $m_{1} \rightarrow m_{1}-1$ в аргументе функции. В терминах символов эта редукция дает

$$
\widetilde{B}\left(m_{1}, m_{2}, m_{3}, z\right)=\widetilde{B}\left(m_{1}-m_{2}, 0, m_{3}, z\right)
$$

что в силу (2.18) возможно, только если $z_{\Re}=-a_{12} / 2$ (мы опускаем тривиальный случай $\left.z_{\Im}=0\right)$. Полагая здесь для простоты

$$
a_{2}=-a_{1}
$$

видим, что данная редукция требует, чтобы символ оператора $B$ был пропорционален дельта-функции $\delta\left(z_{\Re}+a_{1}\right)$, так что вследствие $(2.18)$

$$
\widetilde{B}\left(m_{1}, 0, m_{3}, z\right)=\left(\frac{a_{1}+i z_{\Im}}{a_{1}-i z_{\Im}}\right)^{m_{1}}\left(\frac{a_{3}+i z_{\Im}}{a_{3}-i z_{\Im}}\right)^{m_{3}} b\left(z_{\Im}\right) \delta\left(z_{\Re}+a_{1}\right),
$$

где $b\left(z_{\Im}\right)$ - произвольная функция своего аргумента (данное рассеяния). Оператор $B$ с таким символом, очевидно, удовлетворяет уравнению

$$
a_{13}\left(B^{(1,3)}-B\right)+\left(a_{1}+a_{3}\right)\left(B^{(1)}-B^{(3)}\right)=0,
$$

в то время как соответствующая редукция исходного уравнения (2.2) дает

$$
\begin{aligned}
a_{13}\left(B^{(1,3)}-B\right) & +\left(a_{1}+a_{3}\right)\left(B^{(1)}-B^{(3)}\right)= \\
& =\left[a_{13}\left(B^{(1,3)}-B\right)+\left(a_{1}+a_{3}\right)\left(B^{(1)}-B^{(3)}\right)\right]^{-1} .
\end{aligned}
$$

Обе части этого уравнения не зависят от $m_{1}$, так что (3.4) возникает как результат его суммирования. 
Подчеркнем, что в силу (2.19) символ $\widetilde{K}(m, z)$ оператора одевания является аналитической функцией от $z \in \mathbb{C}$ в полуплоскостях $z_{\Re} \gtrless-a_{1}$ (см. обсуждение в начале этого раздела).

Применяя формулы $(2.7),(2.19),(3.1)$, мы получаем также, что $K^{(2)}=K^{(-1)}$, т. е.

$$
\widetilde{K}\left(m_{1}, m_{2}, m_{3}, z\right)=\widetilde{K}\left(m_{1}-m_{2}, 0, m_{3}, z\right), \quad z \in \mathbb{C} .
$$

Таким образом, уравнение (2.25б) пары Лакса остается неизменным, в то время как для (2.25a) мы имеем

$$
\left(z+2 a_{1}\right) \widetilde{K}^{(-1)}(m, z)=z \widetilde{K}^{(1)}(m, z)+\left(v^{(-1)}(m)-v^{(1)}(m)\right) \widetilde{K}(m, z) .
$$

В силу (2.22) мы также имеем $u\left(m_{1}, m_{2}, m_{3}, z\right)=u\left(m_{1}-m_{2}, 0, m_{3}, z\right)$, а равенство (3.2) дает такую же зависимость функции $v(m)$ от $m_{1}-m_{2}$ и $m_{3}$. В силу этой специфической зависимости от $m$ следует модифицировать определение (2.28) решения Йоста:

$$
\psi\left(m_{1}-m_{2}, m_{3}, \lambda\right)=\widetilde{K}(m, z) z^{m_{1}-m_{2}}\left(z+a_{13}\right)^{m_{3}},
$$

где мы положили

$$
\lambda=z+a_{1},
$$

что на самом деле является символом оператора $A$, см. (2.15). Итак, полагая теперь $m_{2}=0$, мы получаем

$$
v(m) \equiv v\left(m_{1}, m_{3}\right)=v\left(m_{1}, m_{3}, 0\right)-a_{1} m_{1}-a_{3} m_{3},
$$

так что уравнение (1.6в) остается неизменным, $\psi^{(3)}=\psi^{(1)}+\left(v^{(3)}-v^{(1)}\right) \psi$, а (3.5) и сама пара Лакса принимают вид

$$
\begin{aligned}
& \psi^{(1)}=\left(v^{(1)}-v^{(-1)}\right) \psi+\left(\lambda-a_{1}^{2}\right) \psi^{(-1)}, \\
& \psi^{(3)}=\left(v^{(3)}-v^{(-1)}\right) \psi+\left(\lambda-a_{1}^{2}\right) \psi^{(-1)},
\end{aligned}
$$

где $\psi^{(1)}$ во втором равенстве было заменено с использованием (3.9).

Условие совместности для этой пары может быть выведено либо непосредственно, либо как редукция уравнения (1.5) и имеет вид

$$
\left(v^{(1,3)}-v\right)\left(v^{(3)}-v^{(1)}\right)=\left(v^{(-1,3)}-v\right)\left(v^{(3)}-v^{(-1)}\right),
$$

что можно записать как

$$
\left\{\left(v^{(3)}-v^{(-1)}\right)\left(v^{(3)}-v^{(1)}\right)^{(-1)}\right\}^{(1)}=\left(v^{(3)}-v^{(-1)}\right)\left(v^{(3)}-v^{(1)}\right)^{(-1)} .
$$

Итак, оператор умножения в правой (или левой части) равенства имеет символ, не зависящий от $m_{1}$. Принимая во внимание формулу (3.8) и убывание функции $u(m)$ при $m_{1} \rightarrow \infty$, получаем, что в этом пределе

$$
v^{(3)}(m)-v^{( \pm 1)}(m) \rightarrow \pm a_{13} .
$$

Таким образом, уравнение (3.12) дает

$$
\left(v^{(1,3)}-v\right)\left(v^{(3)}-v^{(1)}\right)=a_{3}^{2}-a_{1}^{2},
$$


где мы произвели сдвиг $m_{1} \rightarrow m_{1}+1$. Уравнение (3.14) известно как разностное потенциальное уравнение КдФ. Оно было выведено в работах [17], [18] и подробно обсуждалось в литературе вместе со своими неабелевыми обобщениями (см. работу [19] и цитированную в ней литературу). Мы привели здесь вывод этого уравнения как пример размерной редукции в рамках нашего подхода.

3.2. Редукция $B^{(3)}=B^{(1,2)}$. Эта $(1+1)$-мерная редукция РУХ сохраняет его специфическое свойство: симметрию по независимым переменным. Пусть для простоты

$$
a_{3}=a_{1}+a_{2},
$$

тогда в силу (2.18) эта редукция означает, что $z$ должно удовлетворять условию

$$
\left|z-a_{2}\right|^{2}=a_{1} a_{2}
$$

Другими словами, на окружности (3.16) символ $\widetilde{B}(m, z)$ должен быть пропорционален дельта-функции, так что в данном случае $a_{1} a_{2}>0$ и символ оператора одевания аналитичен внутри и вне окружности (3.16). Заметим также, что в силу этой редукции символы операторов $B$ и $K$ удовлетворяют условиям

$$
\begin{aligned}
\widetilde{B}\left(m_{1}, m_{2}, m_{3}, z\right) & =\widetilde{B}\left(m_{1}+m_{3}, m_{2}+m_{3}, 0, z\right), \\
\widetilde{K}\left(m_{1}, m_{2}, m_{3}, z\right) & =\widetilde{K}\left(m_{1}+m_{3}, m_{2}+m_{3}, 0, z\right),
\end{aligned}
$$

следовательно, с учетом (2.22) мы имеем ту же зависимость $u(m)$ от переменных $m_{i}$, а в силу (2.26) и (3.15) это справедливо и для функции $v$ :

$$
v\left(m_{1}, m_{2}, m_{3}\right)=v\left(m_{1}+m_{3}, m_{2}+m_{3}, 0\right) .
$$

Видно, что при такой редукции уравнение (2.23) не меняется, а (2.24) сводится к

$$
\left(z-a_{2}\right) \widetilde{K}^{(1,2)}(m, z)=z \widetilde{K}^{(1)}(m, z)+\left(v^{(1,2)}(m)-v^{(1)}(m)\right) \widetilde{K}(m, z),
$$

где теперь $m_{3}=0$. Решение Йоста мы вводим посредством равенства (ср. с (2.28))

$$
\psi\left(m_{1}, m_{2}, k\right)=\widetilde{K}\left(m_{1}, m_{2}, 0, z\right) z^{m_{1}}\left(z+a_{12}\right)^{m_{2}},
$$

где

$$
k=\frac{2}{a_{12}}\left(\frac{a_{1}}{z+a_{12}}-\frac{a_{2}}{z}\right)
$$

является спектральным параметром. Наконец, для пары Лакса имеем

$$
\begin{gathered}
\psi^{(2)}-\psi^{(1)}=\left(v^{(2)}-v^{(1)}\right) \psi \\
k \psi^{(1,2)}=\psi^{(1)}+\psi^{(2)}+\left(2 v^{(1,2)}-v^{(1)}-v^{(2)}\right) \psi,
\end{gathered}
$$

а соответствующее нелинейное интегрируемое уравнение записывается как

$$
\left(v^{(1,2)}\left(v^{(2)}-v\right)+v v^{(2)}\right)^{(1)}=\left(v^{(1,2)}\left(v^{(1)}-v\right)+v v^{(1)}\right)^{(2)}
$$

и представляет собой уравнение некоторой $(1+1)$-мерной цепочки с дискретной временной эволюцией, симметричной по обеим независимым переменным. 
3.3. Редукция $B^{(3)}=B^{(-1,-2)}$. Это еще одна редукция, которая также приводит к симметричной цепочке. Повторяя аргументацию, приведенную выше, получаем

$$
\widetilde{B}(m, z)=\widetilde{B}\left(m_{1}-m_{3}, m_{2}-m_{3}, 0, z\right) .
$$

Это означает, что символ $\widetilde{B}(m, z)$ пропорционален дельта-функции на гиперболе, заданной уравнением $3\left(z_{\Re}+a_{1}\right)^{2}-z_{\Im}^{2}=\left(a_{1}+a_{2}\right)^{2}-a_{1} a_{2}$. Опуская другие детали, приведем здесь лишь нелинейное уравнение:

$$
v^{(1,2)}\left(v^{(1)}-v^{(2)}\right)-v^{(-1,-2)}\left(v^{(-1)}-v^{(-2)}\right)=v^{(1)} v^{(-2)}-v^{(-1)} v^{(2)} .
$$

Ниже мы рассмотрим аналогичные редукции на примере высшего РУХ.

\section{4. ВЫСШИЕ РУХ}

Очевидный способ ввести новые дискретные независимые переменные в РУХ увеличить число уравнений эволюции типа (2.1), т. е. ввести в дополнение к разностным переменным $\left\{m_{1}, m_{2}, m_{3}\right\}$ сколь угодно много других переменных $\left\{m_{4}, m_{5}, \ldots\right\}$ так, что динамика по каждой из них задается уравнением $B^{(i)}=\left(A-a_{i}\right) B\left(A-a_{i}\right)^{-1}$, где $a_{4}, a_{5}, \ldots$ - различные (вещественные) параметры. Все эти уравнения эволюции совместны между собой и с исходными уравнениями, но их определение показывает, что для любых $i, j, k$ имеется аналог равенства (2.2) (см. также определение (2.3)):

$$
a_{i j}\left\{B^{(i j)}+B^{(k)}\right\}+\operatorname{cycle}\{i, j, k\}=0 .
$$

Тогда, повторяя построение из п. 2 , получаем, что по любым трем переменным $m_{i}$, $m_{j}$ и $m_{k}$ функция $u\left(m_{1}, \ldots\right)$, заданная в $(2.22)$, и функция $v(m)=u(m)-\sum_{i} a_{i} m_{i}$ (см. (2.26)) удовлетворяют тому же РУХ. Таким образом, это "обобщение" тривиально и может быть интересно только при исследовании симметрий РУХ (см., например, статью [20]).

Итак, для того чтобы получить высшие аналоги РУХ, следует рассмотреть высшие аналоги преобразований подобия (2.1). Пусть $p_{i}=p_{i}(T), i=1,2,3$, являются полиномами от оператора $T$ порядка $n_{i}$ с постоянными коэффициентами, т. е. символы $\tilde{p}_{i}(m, z)=p_{i}(z)$ суть полиномы от $z \in \mathbb{C}$. Будем также считать, что все эти полиномы имеют простые и взаимно различные нули и что коэффициенты при старших степенях равны $I$. Как и ранее, мы рассматриваем оператор $B$ с символом $\widetilde{B}\left(m_{1}, m_{2}, m_{3}, z\right)$, зависящим от разностных переменных $m_{i} \in \mathbb{Z}$, но теперь вместо (2.1) зависимость от этих переменных задается уравнением

$$
B^{(i)}=p_{i} B p_{i}^{-1}, \quad i=1,2,3 .
$$

Заметим, что в силу условия на полиномы $p_{i}$ каждый из них может быть записан в виде

$$
p_{i}(T)=\prod_{j=1}^{n_{i}}\left(T-x_{i j}\right),
$$

так что сдвиг по $i$-й переменной в силу (4.1) эквивалентен $n_{i}$ сдвигам в смысле (2.1). Тем не менее вывод уравнений эволюции (4.1) с помощью таких многомерных редукций крайне сложен даже в линейном случае, поэтому мы рассматриваем построение 
интегрируемых уравнений непосредственно на основе уравнения (4.1). Для согласования с операторным подходом, развитым в п. 2.2, мы выбираем

$$
p_{1}(T)=T
$$

Оператор одевания $K$ определяется той же $\bar{\partial}$-задачей (2.19), а его зависимость от $m_{i}$ задается уравнением (4.1). Тогда, как и ранее, если предположить однозначную разрешимость задачи (2.19), существуют полиномы $P_{i}(T)$, такие что

$$
K^{(i)} p_{i}=P_{i} K, \quad i=1,2,3
$$

Запишем равенства

$$
p_{i}(T)=\sum_{j=0}^{n_{i}} y_{i j} T^{j}, \quad P_{i}(T)=\sum_{j=0}^{n_{i}} Y_{i j} T^{j}
$$

где $y_{i, n_{i}}=Y_{i, n_{i}} \equiv 1$ и все $y_{i j}$ суть константы, в то время как $Y_{i j}$ - операторы умножения, $\widetilde{Y}_{i j}(m, z)=\widetilde{Y}_{i j}(m)$. Тогда (4.4) принимает вид

$$
K^{(i)} \sum_{j=0}^{n_{i}} y_{i j} T^{j}=\sum_{j=0}^{n_{i}} Y_{i j} K^{(1 \times j)} T^{j}, \quad i=1,2,3 .
$$

Здесь мы ввели обозначение (ср. с (2.11))

$$
\widetilde{K}^{(1 \times j)}\left(m_{1}, m_{2}, \ldots, z\right)=\widetilde{K}\left(m_{1}+j, m_{2}, \ldots, z\right) .
$$

Уравнение (4.6) можно упростить, записав в терминах решения Йоста (ср. с (2.28))

$$
\varphi(m, z)=\widetilde{K}(m, z) p_{1}(z)^{m_{1}} p_{2}(z)^{m_{2}} p_{3}(z)^{m_{3}},
$$

что вследствие $(2.8),(4.6)$ и (4.7) дает

$$
\varphi^{(i)}\left(m_{1}, m_{2}, m_{3}, z\right)=\sum_{j=0}^{m_{i}} Y_{i, j}(m) \varphi\left(m_{1}+j, m_{2}, m_{3}, z\right) .
$$

Представление символа оператора $B$ вытекает из (4.1) по аналогии с (2.17):

$$
\widetilde{B}(m, z)=\oint_{|\zeta|=1} \frac{d \zeta}{2 \pi i \zeta} \zeta^{m_{1}}\left(\frac{p_{2}(\zeta z)}{p_{2}(z)}\right)^{m_{2}}\left(\frac{p_{3}(\zeta z)}{p_{3}(z)}\right)^{m_{3}} b(\zeta, z),
$$

где $b(\zeta, z)$ - некоторая функция. Чтобы не допустить роста этого символа по $m_{2}$ или $m_{3}$, мы налагаем условие $\left|p_{i}(\zeta z)\right|=\left|p_{i}(z)\right|$. Более того, для простоты примем, что полиномы $p_{i}(z)$ имеют вещественные коэффициенты и $b(\zeta, z)=\delta_{c}(\zeta z / \bar{z}) f(z)$, где $\delta_{c}(\zeta)$ - дельта-функция на единичном контуре, а $f(z)$ - произвольная функция переменной $z \in \mathbb{C}$. Тогда по аналогии с (2.18) мы получаем представление

$$
\widetilde{B}(m, z)=\left(\frac{\bar{z}}{z}\right)^{m_{1}}\left(\frac{p_{2}(\bar{z})}{p_{2}(z)}\right)^{m_{2}}\left(\frac{p_{3}(\bar{z})}{p_{3}(z)}\right)^{m_{3}} f(z) .
$$


Повторяя рассуждения из п. 2.2, мы выводим из предположения об однозначной разрешимости задачи (2.19), что уравнения эволюции (4.4) (или (4.6)) совместны:

$$
K^{(i, j)}=K^{(j, i)}
$$

при любых $i$ и $j$. Эта совместность позволяет вывести дискретный аналог системы Захарова-Шабата [21]. Действительно, благодаря соотношениям (2.16) и (4.4)

$$
K^{(i, j)} p_{i} p_{j}=P_{i}^{(j)} K^{(j)} p_{j}=P_{i}^{(j)} P_{j} K, \quad i, j=1,2,3 .
$$

Принимая во внимание, что полиномы $p_{i}$ и $p_{j}$ с постоянными коэффициентами коммутируют (см. равенство (2.10)), мы получаем, что в силу (4.11) левая часть уравнения (4.12) симметрична по $i$ и $j$. Тогда правая часть дает

$$
P_{i}^{(j)} P_{j}=P_{j}^{(i)} P_{i}, \quad i, j=1,2,3 .
$$

Обратно, уравнение (4.11) следует из (4.13).

Дискретная версия (4.13) системы Захарова-Шабата позволяет вывести уравнения эволюции для коэффициентных функций полиномов $P_{i}$. Во-первых, если в (4.13) индекс $i$ или $j$ равен единице, то это равенство превращается в тождество в силу $(2.11)$ и $(4.3)$ (ср. с (2.21)). Пусть теперь $i, j \neq 1$. Подставляя тогда $P_{i}(T)$ из (4.5), перемещаем все операторы $T$ направо с помощью соотношения (2.11). Тогда очевидно, что обе части равенства (4.13) представляют собой полиномы от $T$ порядка $n_{2}+n_{3}$ с единичными старшими коэффициентами. Приравнивая коэффициенты при равных степенях оператора $T$, мы получаем в точности $n_{2}+n_{3}$ уравнений

$$
\sum_{k=\max \left\{l-n_{j}, 0\right\}}^{\min \left\{l, n_{i}\right\}}\left(Y_{i k}^{(j)} Y_{i k}^{(1 \times k)}-Y_{j, m-k}^{(i)} Y_{i k}^{(1 \times(l-k))}\right)=0, \quad l=0,1, \ldots, n_{2}+n_{3}-1,
$$

где использовано обозначение (4.7); уравнение для $l=n_{2}+n_{3}$ опущено ввиду его тривиальности. С другой стороны, необходимо отметить, что функции $Y_{i j}$ не являются независимыми, поскольку благодаря (4.6) эти $n_{2}+n_{3}$ коэффициентных функций выражаются через $n=\max \left\{n_{2}, n_{3}\right\}$ коэффициентных функции асимптотического разложения оператора одевания

$$
K=I+\sum_{j=1}^{n} k_{j} T^{-j}+o\left(T^{-n}\right)
$$

где символы операторов $k_{j}$ не зависят от $z, \tilde{k}_{j}(m, z)=k_{j}(m)$, и где $o\left(T^{-n}\right)$ понимается в смысле символов, т. е. как $o\left(z^{-n}\right)$. По построению система (4.14) остается совместной и дает нелинейную интегрируемую систему.

Простейший пример такой интегрируемой системы получается при $n_{2}=n_{3}=1$, т. е. при $P_{2}=T+Y_{2,0}, P_{3}=T+Y_{3,0}$. Тогда (4.14) сводится к системе

$$
Y_{2,0}^{(1)}+Y_{3,0}^{(2)}=Y_{3,0}^{(1)}+Y_{2,0}^{(3)}, \quad Y_{3,0}^{(2)} Y_{2,0}=Y_{2,0}^{(3)} Y_{3,0}
$$

Если ввести (ср. с соотношениями $(2.23),(2.24)$ и $(2.26))$ операторы $Y_{2,0}=v^{(2)}-v^{(1)}$ и $Y_{3,0}=v^{(3)}-v^{(1)}$, то первое уравнение в (4.16) превращается в тождество, а второе дает в точности РУХ (1.5). 
ЗАмЕчАниЕ. В описанном выше построении мы нигде не меняли порядок коэффициентов $y_{i j}$ и $Y_{i j}$. Это означает, что соотношение (4.13) справедливо также в неабелевом случае, когда эти коэффициенты не являются взаимно коммутирующими [14].

\section{5. ПРИМЕР ВЫСШЕГО РУХ}

Расмотрим пример высшего уравнения, наиболее близкого к РУХ. Пусть динамика оператора $B$ в (4.1) задается посредством полиномов

$$
p_{1}(T)=T, \quad p_{2}(T)=T+a_{12}, \quad p_{3}(T)=\left(T+a_{1}\right)^{2}-a_{3}^{2},
$$

где было учтено (4.3) и где $a_{1}, a_{2}$ и $a_{3}^{2}$ - вещественные константы, $a_{12}=a_{1}-a_{2} \neq 0$, $a_{3} \neq 0, \pm a_{2}$. Обозначим первую разность операторов как $\Delta_{i} B=B^{(i)}-B$. Тогда $B$ удовлетворяет разностному уравнению

$$
\begin{aligned}
& {\left[\left(\Delta_{1} a_{1}-\Delta_{2} a_{2}\right)^{2}-a_{3}^{2}\left(\Delta_{1}-\Delta_{2}\right)^{2}\right] \Delta_{3} B=} \\
& \quad=a_{12} \Delta_{1} \Delta_{2}\left(a_{12} \Delta_{1} \Delta_{2}+2 \Delta_{1} a_{1}-2 \Delta_{2} a_{2}\right) B
\end{aligned}
$$

которое следует из соответствующего коммутаторного тождества. Его также можно проверить непосредственно, поскольку в данном случае (4.10) принимает вид

$$
\widetilde{B}(m, z)=\left(\frac{\bar{z}}{z}\right)^{m_{1}}\left(\frac{\bar{z}+a_{12}}{z+a_{12}}\right)^{m_{2}}\left(\frac{\left(\bar{z}+a_{1}\right)^{2}-a_{3}^{2}}{\left(z+a_{1}\right)^{2}-a_{3}^{2}}\right)^{m_{3}} f(z) .
$$

Оператор одевания $K$, как всегда, определяется из (2.19), так что в силу (4.5)

$$
\begin{aligned}
& P_{2}(T)=T+Y_{20}, \\
& P_{3}(T)=T^{2}+Y_{31} T+Y_{30},
\end{aligned}
$$

где символы операторов $Y_{i j}$ не зависят от $z$. Тогда с учетом (4.6) пара Лакса записывается как

$$
\begin{aligned}
K^{(2)}\left(T+a_{12}\right) & =K^{(1)} T+Y_{20} K, \\
K^{(3)}\left[\left(T+a_{1}\right)^{2}-a_{3}^{2}\right] & =K^{(1,1)} T^{2}+Y_{31} K^{(1)} T+Y_{30} K,
\end{aligned}
$$

где вследствие (4.13) и (5.4), (5.5) коэффициенты удовлетворяют уравнениям

$$
\begin{aligned}
Y_{31}^{(1)}+Y_{20}^{(3)} & =Y_{20}^{(1,1)}+Y_{31}^{(2)}, \\
Y_{30}^{(1)}+Y_{20}^{(3)} Y_{31} & =Y_{30}^{(2)}+Y_{31}^{(2)} Y_{20}^{(1)}, \\
Y_{20}^{(3)} Y_{30} & =Y_{30}^{(2)} Y_{20} .
\end{aligned}
$$

Учитывая симметрию этой редукции по переменным $m_{1}$ и $m_{2}$, естественно переписать (5.7) с помощью (5.6) в явно симметричной форме. Тогда для пары Лакса мы получаем

$$
\begin{aligned}
K^{(2)}\left(A-a_{2}\right)= & K^{(1)}\left(A-a_{1}\right)+Y_{20} K, \\
K^{(3)}\left[A^{2}-a_{3}^{2}\right]= & K^{(1,2)}\left(A-a_{1}\right)\left(A-a_{2}\right)+ \\
& +X_{31}\left(K^{(1)}\left(A-a_{1}\right)+K^{(2)}\left(A-a_{2}\right)\right)+X_{30} K,
\end{aligned}
$$


где снова для симметрии мы воспользовались равенством (2.14), а новые коэффициенты равны

$$
X_{31}=\frac{1}{2}\left(Y_{31}-Y_{20}^{(1)}\right), \quad X_{30}=Y_{30}+X_{31} Y_{20} .
$$

В этих терминах соотношения (5.8) также принимают симметричный вид:

$$
\begin{aligned}
Y_{20}^{(3)} & =Y_{20}^{(1,2)}+2 X_{31}^{(2)}-2 X_{31}^{(1)} \\
2 Y_{20}^{(3)} X_{31} & =X_{20}^{(2)}-X_{30}^{(1)}+X_{31}^{(2)} Y_{20}^{(2)}+X_{31}^{(1)} Y_{20}^{(1)} \\
2 Y_{20}^{(3)} X_{30} & =\left[X_{30}^{(2)}+X_{30}^{(1)}+X_{31}^{(2)} Y_{20}^{(2)}-X_{31}^{(1)} Y_{20}^{(1)}\right] Y_{20} .
\end{aligned}
$$

Коэффициенты $Y_{i j}$ (или $X_{i j}$ ) должны определяться путем подстановки асимптотического разложения (4.15) в (5.6), (5.7) или в (5.9), (5.10). Для того чтобы сохранить ранее отмеченную симметрию, мы используем два последних уравнения, и, как подсказывает (2.14), вместо (4.15) запишем разложение в виде

$$
K=I+u A^{-1}+w A^{-2}+\cdots,
$$

где символы операторов $u$ и $w$ зависят лишь от переменных $m$. Мы опускаем здесь детали вычислений, а для того чтобы представить их результаты, вводим функции

$$
\begin{aligned}
v(m)= & u(m)-m_{1} a_{1}-m_{2} a_{2}, \\
f(m)= & w(m)-\left(m_{1} a_{1}+m_{2} a_{2}\right) u(m)+ \\
& +\frac{1}{2}\left(m_{1} a_{1}+m_{2} a_{2}\right)^{2}-\frac{m_{1} a_{1}^{2}}{2}-\frac{m_{2} a_{2}^{2}}{2}-m_{3} a_{3}^{2} .
\end{aligned}
$$

Тогда, подставляя $(5.15)$ в $(5.9)$ и $(5.10)$, получаем

$$
\begin{gathered}
Y_{20}=v^{(2)}-v^{(1)}, \\
f^{(2)}-f^{(1)}=Y_{20} v, \\
X_{31}=\frac{1}{2}\left(v^{(3)}-v^{(1,2)}\right), \\
X_{30}=f^{(3)}-f^{(1,2)}-X_{31}\left(v^{(1)}+v^{(2)}\right) .
\end{gathered}
$$

Итак, три функции $Y_{20}, X_{30}$ и $X_{31}$ выражаются через две функции $v$ и $f$ и должны удовлетворять трем уравнениям (5.12)-(5.14). Как мы отмечали выше, эта система совместна. В частности, легко проверить, что (5.12) и (5.13) превращаются в тождества в силу уравнений (5.18a)-(5.18в), а (5.18г) сводится к

$$
\begin{gathered}
2 v^{(2,3)}\left[f^{(3)}-f^{(1,2)}-v^{(3)} v^{(2)}+v^{(2)} v^{(1,2)}\right]- \\
-2 v^{(1,3)}\left[f^{(3)}-f^{(1,2)}-v^{(3)} v^{(1)}+v^{(1)} v^{(1,2)}\right]= \\
=\left(v^{(2)}-v^{(1)}\right)\left[\left(f^{(2)}-v^{(2)} v\right)^{(3)}+\left(f^{(1)}-v^{(1)} v\right)^{(3)}-\right. \\
\left.-\left(f^{(2)}-v^{(2)} v\right)^{(1,2)}-\left(f^{(1)}-v^{(1)} v\right)^{(1,2)}\right],
\end{gathered}
$$

что дает одно уравнение на две функции. Эти функции не являются независимыми, поскольку вследствие (5.18а) и (5.18б)

$$
f^{(2)}-v^{(2)} v=f^{(1)}-v^{(1)} v .
$$


Уравнения (5.19) и (5.20) - это уравнения интегрируемой системы, дающие пример высшего РУХ. Она получается как условие совместности пары Лакса (5.9), (5.10), которая в терминах решения Йоста (ср. с (4.8))

$$
\varphi(m, z)=\widetilde{K}(m, z) z^{m_{1}}\left(z+a_{12}\right)^{m_{2}}\left[\left(z+a_{1}\right)^{2}-a_{3}^{2}\right]^{m_{3}}
$$

записывается как

$$
\begin{aligned}
\varphi^{(2)}= & \varphi^{(1)}+\left(v^{(2)}-v^{(1)}\right) \varphi \\
\varphi^{(3)}= & \varphi^{(1,2)}+\left(v^{(3)}-v^{(1,2)}\right) \frac{\varphi^{(1)}+\varphi^{(2)}}{2}+ \\
& +\left[f^{(3)}-f^{(1,2)}-\frac{1}{2}\left(v^{(1)}+v^{(2)}\right)\left(v^{(3)}-v^{(1,2)}\right)\right] \varphi
\end{aligned}
$$

где было использовано (5.18). Опуская детали, отметим, что в силу (5.22) уравнение (5.23) принимает вид

$$
\varphi^{(3)}=\varphi^{(1,1)}+\left(v^{(3)}-v^{(1,1)}\right) \varphi^{(1)}+\left[f^{(3)}-f^{(1,2)}-v^{(1)}\left(v^{(3)}-v^{(1,2)}\right)\right] \varphi
$$

и вместе с (5.22) дает эквивалентную пару Лакса.

\section{6. РЕДУКЦИИ СИСТЕМЫ (5.19), (5.20)}

6.1. Редукция $B^{(3)}=B$. Система (5.19), (5.20) также допускает $(1+1)$-мерные редукции, и обсуждение в начале п. 3 применимо здесь с той лишь разницей, что кривая на плоскости $\mathbb{C}$ теперь определяется из (5.3) вместо (2.18). В силу этого, несмотря на то что система (5.19), (5.20) отличается от РУХ только зависимостью от переменной $m_{3}$, в данном случае редукция $B^{(3)}=B$ нетривиальна. Действительно, с учетом (5.3) эта редукция означает, что символ $\widetilde{B}(m, z)$ отличен от нуля, если $\left(\bar{z}+a_{1}\right)^{2}=\left(z+a_{1}\right)^{2}$, т. е. $z_{\Re}=-a_{1}$, так что функция $f(z)$ в (5.3) должна быть пропорциональна $\delta\left(z_{\Re}+a_{1}\right)$ :

$$
\widetilde{B}(m, z)=\left(\frac{a_{1}+i z_{\Im}}{a_{1}-i z_{\Im}}\right)^{m_{1}}\left(\frac{a_{1}+i z_{\Im}}{a_{1}-i z_{\Im}}\right)^{m_{2}} \delta\left(z_{\Re}+a_{1}\right) r\left(z_{\Im}\right) .
$$

Тогда обратная задача (2.19) показывает, не только что оператор одевания не зависит от $m_{3}$, но и что его символ $\widetilde{K}\left(m_{1}, m_{2}, z\right)$ - аналитическая функция от $z$, когда $z_{\Re} \neq-a_{1}$.

Чтобы получить редуцированные пару Лакса и нелинейное уравнение, отметим, что коэффициенты асимптотического разложения (5.15) не зависят от переменной $m_{3}$, т. е. $u(m)=u\left(m_{1}, m_{2}\right), w(m)=w\left(m_{1}, m_{2}\right)$. Соответственно, в силу (5.16) и (5.17) $v(m)=v\left(m_{1}, m_{2}\right), f(m)=g\left(m_{1}, m_{2}\right)-a_{3}^{3} m_{3}$, где $g\left(m_{1}, m_{2}\right)=f\left(m_{1}, m_{2}, 0\right)$. Подставляя эти равенства в (5.19) и (5.20), мы получаем нелинейную интегрируемую систему

$$
\begin{gathered}
\left(g^{(2)}-v^{(2)} v\right)^{(1,2)}+\left(g^{(1)}-v^{(1)} v\right)^{(1,2)}-\left(g^{(2)}-v^{(2)} v\right)^{(1)}-\left(g^{(1)}-v^{(1)} v\right)^{(2)}- \\
-\left(g^{(2)}-v^{(2)} v\right)-\left(g^{(1)}-v^{(1)} v\right)+2 g-2 v\left(v^{(1)}+v^{(2)}\right)=0 \\
g^{(2)}-v^{(2)} v=g^{(1)}-v^{(1)} v .
\end{gathered}
$$


Учитывая, что теперь символ $\widetilde{K}(m, z)$ не зависит от $m_{3}$, определим решения Йоста посредством равенства (см. (5.21))

$$
\psi\left(m_{1}, m_{2}, z+a_{1}\right)=\widetilde{K}(m, z) z^{m_{1}}\left(z+a_{12}\right)^{m_{2}} \equiv \frac{\varphi(m, z)}{\left[\left(z+a_{1}\right)^{2}-a_{3}^{2}\right]^{m_{3}}} .
$$

В результате из (5.23), (5.21) получаем редуцированную пару Лакса

$$
\begin{gathered}
\psi^{(1,1)}+\left(v-v^{(1,1)}\right) \psi^{(1)}+\left[g-g^{(1,2)}-v^{(1)}\left(v-v^{(1,2)}\right)\right] \psi=\lambda^{2} \psi, \\
\psi^{(2)}=\psi^{(1)}+\left(v^{(2)}-v^{(1)}\right) \psi
\end{gathered}
$$

где использован спектральный параметр $\lambda=z+a_{1}$, см. (3.7).

6.2. Редукция $B^{(2)}=B^{(-1)}$. Эта редукция полностью аналогична рассмотренной в п. 3.1. Мы снова имеем равенство (3.1), что в силу (5.3) означает, что в данном случае $\widetilde{B}(m, z) \sim \delta\left(z_{\Re}+a_{12} / 2\right)$, где $a_{12}=a_{1}-a_{2}$, т. е.

$$
\widetilde{B}(m, z)=\left(\frac{a_{12} / 2+i z_{\Im}}{a_{12} / 2-i z_{\Im}}\right)^{m_{1}-m_{2}}\left(\frac{\left(\left(a_{1}+a_{2}\right) / 2-i z_{\Im}\right)^{2}-a_{3}^{2}}{\left(\left(a_{1}+a_{2}\right) / 2+i z_{\Im}\right)^{2}-a_{3}^{2}}\right)^{m_{3}} \delta\left(z_{\Re}+a_{12} / 2\right) r\left(z_{\Im}\right) .
$$

Символ оператора одевания аналитичен, если $z_{\Re} \neq-a_{12} / 2$, кроме того, мы имеем $\widetilde{K}(m, z)=\widetilde{K}\left(m_{1}-m_{2}, 0, m_{3}, z\right)$ и $u(m)=u\left(m_{1}-m_{2}, 0, m_{3}\right), w(m)=w\left(m_{1}-m_{2}, 0, m_{3}\right)$, но здесь мы не используем условие (3.2), чтобы сохранить зависимость от $m_{3}$. Соответственно, в (5.16) и (5.17) нужно использовать функции $u(m)$ и $w(m)$, но не $v(m)$ и $f(m)$. Решение Йоста определяется как

$$
\psi\left(m_{1}-m_{2}, m_{3}, z+a_{1}\right)=\widetilde{K}(m, z) z^{m_{1}}\left[\left(z+a_{1}\right)^{2}-a_{3}^{2}\right]^{m_{3}} \equiv \frac{\varphi(m, z)}{\left[z\left(z+a_{12}\right)\right]^{m_{2}}},
$$

так что первое уравнение пары Лакса, как следует из (5.23) и (5.16), есть

$$
\psi^{(1,1)}-\left(u^{(1,1)}-u+a_{12}\right) \psi^{(1)}=\left(\lambda-a_{1}\right)\left(\lambda-a_{2}\right) \psi
$$

Мы опускаем дальнейшее построение, поскольку оно является очевидным, излишне сложным и неинформативным.

6.3. Редукция $B^{(3)}=B^{(1,2)}$. Аналогично п. 3.2 мы теперь имеем соотношение (3.17), которое в силу (5.3) означает, что, для того чтобы удовлетворить уравнению этой редукции и сохранить зависимость от $m_{1}$ и $m_{2}$, символ $\widetilde{B}(m, z)$ должен иметь носитель на окружности $\left(a_{1}+a_{2}\right)\left|z+a_{1}\right|^{2}-2\left(a_{3}^{2}+a_{1} a_{2}\right)\left(z+a_{1}\right) \Re=\left(a_{1}+a_{2}\right) a_{3}^{2}$ (ср. с (3.16)). Мы полагаем, что $a_{1}+a_{2} \neq 0$. Пусть для упрощения

$$
a_{3}^{2}=-a_{1} a_{2}<0,
$$

в результате для символа оператора $B$ получаем представление

$$
\widetilde{B}(m, z)=\left(\frac{\bar{z}}{z}\right)^{m_{1}+m_{3}}\left(\frac{\bar{z}+a_{12}}{z+a_{12}}\right)^{m_{2}+m_{3}} \delta\left(\left|z+a_{1}\right|^{2}-a_{1} a_{2}\right) r(z) .
$$

Функции $u(m)$ и $w(m)$ как коэффициенты разложения (5.15) символа $\widetilde{K}$ также удовлетворяют уравнениям $u^{(3)}(m)=u^{(1,2)}(m), w^{(3)}(m)=w^{(1,2)}(m)$, так что вследствие (5.17) функция $w(m)$ выпадает из уравнения $(5.18 г)$, эволюционного уравнения (5.19) и пары Лакса $(5.24),(5.22)$. С учетом зависимости оператора одевания от 
переменных $m, \widetilde{K}(m, z)=\widetilde{K}\left(m_{1}+m_{3}, m_{2}+m_{3}, 0, z\right)$, решение Йоста должно быть определено как (ср. с (5.21) и (6.4))

$$
\begin{aligned}
\psi\left(m_{1}+m_{3}, m_{2}+m_{3}, z+a_{1}\right) & =\widetilde{K}(m, z) z^{m_{1}+m_{3}}\left(z+a_{12}\right)^{m_{2}+m_{3}} \equiv \\
& \equiv\left(\frac{z\left(z+a_{12}\right)}{\left(z+a_{1}\right)^{2}-a_{3}^{2}}\right)^{m_{3}} \varphi(m, z),
\end{aligned}
$$

и пара Лакса принимает вид

$$
\begin{aligned}
k \psi^{(1,1)} & =\left(1-k\left(q^{(2)}-q^{(1)}\right)^{(1)}\right) \psi^{(1)}+\left(q^{(2)}-q\right)^{(1)} \psi, \\
\psi^{(2)} & =\psi^{(1)}+\left(q^{(2)}-q^{(1)}\right) \psi,
\end{aligned}
$$

где теперь спектральный параметр $k$ определен как

$$
k=\frac{z+a_{1}}{z\left(z+a_{12}\right)} .
$$

В (6.11), (6.12) мы также ввели новую зависимую переменную (ср. с (5.16))

$$
q\left(m_{1}, m_{2}\right)=u\left(m_{1}, m_{2}\right)+m_{1} a_{2}+m_{2} a_{1} .
$$

С учетом этого обозначения уравнение (5.19) при данной редукции дает

$$
\left(q^{(2)}-q\right)^{(1,2)}\left(q^{(1)}-q\right)^{(2)}=\left(q^{(1)}-q\right)^{(1,2)}\left(q^{(2)}-q\right)^{(1)}
$$

и легко проверить, что это нелинейное уравнение является условием совместности для пары Лакса (6.11), (6.12). Подобно (3.23) это уравнение симметрично по обеим независимым переменным.

\section{7. ЗАКЛЮЧЕНИЕ}

Мы рассмотрели метод вывода нелинейных (разностных) интегрируемых уравнений и их пар Лакса. Наше построение не свободно от предположений, прежде всего от предположений об однозначной разрешимости $\bar{\partial}$-задачи (2.19) и о существовании асимптотических разложений (2.22) и (4.15). Эти предположения были крайне существенны для нашего вывода. С другой стороны, когда пары Лакса получены, вывод условия их совместности является чисто алгебраической процедурой, которая не нуждается ни в каких предположениях и приводит к нелинейному интегрируемому уравнению. Например, высшее РУХ, т. е. система (5.19), (5.20), есть условие совместности уравнений (5.22), (5.23), что можно проверить непосредственно.

В работах [12]--[14] и [16] мы отмечали, что интегрируемость нелинейных уравнений напрямую связана с существованием коммутаторных тождеств типа (2.4) для РУХ и типа (5.2) для его высшего аналога, рассмотренного в разделе (5). Эти тождества приводят к существованию линейных уравнений для оператора $B$, порожденных либо преобразованиями подобия типа (2.15), (4.1) или (5.1) в нашем случае, либо коммутаторными тождествами в случае дифференциальных операторов. Во всех рассмотренных случаях эти преобразования задаются с помощью функций от оператора сдвига $T$ (оператора дифференцирования $D$ для дифференциальных уравнений). Однако специфическим свойством выведенных линейных 
уравнений является то, что они не содержат оператор $T$ (см. (2.2) и (5.4)). Это гарантирует существование линеаризованных версий данных интегрируемых уравнений. Действительно, обратная задача (2.19), линеаризованная по оператору $B$, дает в силу (2.22)

$$
u(m)=-\frac{1}{\pi^{2}} \int d^{2} z \widetilde{B}(m, z)+\cdots,
$$

что доказывает данное утверждение.

В общем случае (4.1) существование соответствующего коммутаторного тождества эквивалентно существованию полинома $Q\left(x_{1}, x_{2}, x_{3}\right)$, такого что

$$
Q\left(\operatorname{Ad}_{1}, \operatorname{Ad}_{2}, \operatorname{Ad}_{3}\right)=0,
$$

где мы обозначили присоединенное действие оператора $T$ на ассоциативной алгебре, рассматривавшейся во введении, как

$$
\operatorname{Ad}_{i} B=p_{i}(T) B p_{i}(T)^{-1}, \quad i=1,2,3 .
$$

Здесь $B$ - произвольный элемент этой алгебры, но, если мы включаем его зависимость от переменных $m_{i}$ посредством уравнения (4.1), $B^{(i)}=\operatorname{Ad}_{i} B$, мы получаем в силу (7.2) замкнутое линейное уравнение для оператора $B\left(m_{1}, m_{2}, m_{3}\right)$ (cp. с (5.2)). Эти соображения и построения, представленные в работе, показывают, что естественно предположить, что единственными линейными разностными уравнениями в размерности $(2+1)$, которые можно обобщить до нелинейных интегрируемых уравнений, являются те, которые можно представить в виде коммутаторных тождеств. Заметим, что в этих рассуждениях мы не используем равенство (4.3).

Если выбраны три полинома $p_{i}$, то проблема существования соответствующего коммутаторного тождества сводится к проблеме существования полинома $Q$ от трех переменных такого, что для любых $x$ и $y$ из $\mathbb{C}$ справедливо равенство

$$
Q\left(\frac{p_{1}(x)}{p_{1}(y)}, \frac{p_{2}(x)}{p_{2}(y)}, \frac{p_{3}(x)}{p_{3}(y)}\right) \equiv 0 .
$$

Хотя решение этой проблемы нам неизвестно, тот факт, что наше построение в разделе 4 не встретило препятствий, показывает возможность существования его решения. Конечно, такое рассуждение не является доказательством, поскольку наш подход содержал предположения, которые весьма существенны для него. С другой стороны, коммутаторные тождества должны существовать в более общей ситуации, когда вместо полиномов $p_{i}$ мы имеем дело с мероморфными функциями и когда их коэффициенты не коммутируют с $B$ (неабелев случай, см., например, [15]).

\section{Список литературы}

[1] R. Hirota, "Nonlinear partial difference equations. II. Discrete-time Toda equations", J. Phys. Soc. Japan, 43:6 (1977), 2074-2078.

[2] R. Hirota, "Discrete analogue of a generalized Toda equation", J. Phys. Soc. Japan, 50:11 (1981), 3785-3791.

[3] T. Miwa, "On Hirota's difference equation", Proc. Japan Acad. Ser. A, 58:1 (1982), 9-12.

[4] L. V. Bogdanov, B. G. Konopelchenko, "Analytic-bilinear approach to integrable hierarchies. I. Generalized KP hierarchy", J. Math. Phys., 39:9 (1998), 4683-4700; "Analytic-bilinear approach to integrable hierarchies. II. Multicomponent KP and 2D Toda lattice hierarchies", 4701-4728. 
[5] А. В. Забродин, "Разностные уравнения Хироты", ТМФ, 113:2 (1997), 179-230.

[6] А. В. Забродин, "Преобразования Беклунда для разностного уравнения Хироты и суперсимметричный анзац Бете", ТМФ, 155:1 (2008), 74-93, arXiv: 0705.4006.

[7] S. Saito, "Octahedral structure of the Hirota-Miwa equation", J. Nonlinear Math. Phys., 19:4 (2012), 1250032, 12 pp.

[8] J. J. C. Nimmo, "On a non-Abelian Hirota-Miwa equation", J. Phys. A: Math. Gen., 39:18 (2006), 5053-5065.

[9] C. R. Gibson, J. J. C. Nimmo, Y. Ohta, "Quazideterminant solutions of a non-Abelian Hirota-Miwa equation", J. Phys. A: Math. Theor., 40:42 (2007), 12607-12617, arXiv: nlin/0702020.

[10] A. Doliwa, "The affine Weyl group symmetry of Desargues maps and of the non-commutative Hirota-Miwa system", Phys. Lett. A, 375:9 (2011), 1219-1224, arXiv: 1006.3380.

[11] I. Krichever, P. Wiegmann, A. Zabrodin, "Elliptic solutions to difference non-linear equations and related many-body problems", Commun. Math. Phys., 193:2 (1998), 373-396, arXiv: hep-th/9704090.

[12] А.К. Погребков, "Коммутаторные тождества на ассоциативных алгебрах и интегрируемость нелинейных эволюционных уравнений”, ТМФ, 154:3 (2008), 477-491.

[13] A. K. Pogrebkov, "Hirota difference equation and a commutator identity on an associative algebra", Алгебра и анализ, 22:3 (2010), 191-205.

[14] А.К. Погребков, "Коммутаторные тождества на ассоциативных алгебрах, разностное неабелево уравнение Хироты и его редукции", ТМФ, 187:3 (2016), 433-446.

[15] V.E. Zakharov, E. I. Schulman, "Degenerative dispersion laws, motion invariants and kinetic equations", Phys. D, 1:2 (1980), 192-202.

[16] A. K. Pogrebkov, "2D Toda chain and associated commutator identity", Geometry, Topology, and Mathematical Physics. S. P. Novikov's Seminar: 2006-2007, Amer. Math. Soc. Transl. Ser. 2, 224, eds. V. M. Buchstaber, I. M. Krichever, AMS, Providence, RI, 2008, 261-270.

[17] F. W. Nijhoff, H. W. Capel, G. L. Wiersma, G. R. W. Quispel, "Bäcklund transformations and three-dimensional lattice equations", Phys. Lett. A, 105:6 (1984), 267-272.

[18] F. Nijhoff, H. Capel, "The discrete Korteweg-de Vries equation", Acta Appl. Math., 39:1-3 (1995), 133-158.

[19] S. Konstantinou-Rizos, T. E. Kouloukas, "A noncommutative discrete potential KdV lift", J. Math. Phys., 59:6 (2018), 063506, 13 pp.

[20] A. K. Pogrebkov, "Symmetries of the Hirota difference equation", SIGMA, 13 (2017), 053, $14 \mathrm{pp}$.

[21] В.Е. Захаров, А. Б. Шабат, "Схема интегрирования нелинейных уравнений математической физики методом обратной задачи рассеяния. I", Функи. анализ и его прил., 8:3 (1974), 43-53. 